\title{
GENERATION OF TWO-DIMENSIONAL WATER WAVES BY MOVING BOTTOM DISTURBANCES
}

\author{
HAYK NERSISYAN, DENYS DUTYKH*, AND ENRIQUE ZUAZUA
}

\begin{abstract}
In this study we investigate the potential and limitations of the wave generation by disturbances moving at the bottom. More precisely, we assume that the wavemaker is composed of an underwater object of a given shape which can be displaced according to a prescribed trajectory. The practical question we address in this study is how to compute the wavemaker shape and its trajectory in order to generate a wave with prescribed characteristics? For the sake of simplicity we model the hydrodynamics by a generalized forced Benjamin-Bona-Mahony (BBM) equation. This practical problem is reformulated as a constrained nonlinear optimization problem. Additional constraints are imposed in order to fulfill various practical design requirements. Finally, we present some numerical results in order to demonstrate the feasibility and performance of the proposed methodology.
\end{abstract}

\section{Contents}

1 Introduction 1

2 Mathematical model $\quad 4$

3 Well-posedness of the gBBM equation $\quad 6$

3.1 Optimization problem 8

4 Numerical results $\quad 9$

5 Conclusions $\quad 18$

\section{Introduction}

The problem of wave generation is complex and has many practical applications. On the scale of a laboratory wave tank a traditional wavemaker is composed of numerous paddles attached to a vertical wall and which can move independently according to a prescribed program. These wavemakers have been successfully used to conduct laboratory experiments at least since late 60's [27, 39].

In this study we investigate theoretically and numerically the potential for practical applications of a different kind of wave making devices. Namely, the mechanism considered hereinbelow is composed mainly of an underwater object which can be displaced along a portion of the bottom with the prescribed trajectory. In mathematical terms, we study the

Key words and phrases. wave generation; moving bottom; BBM equation; optimization.

* Corresponding author. 


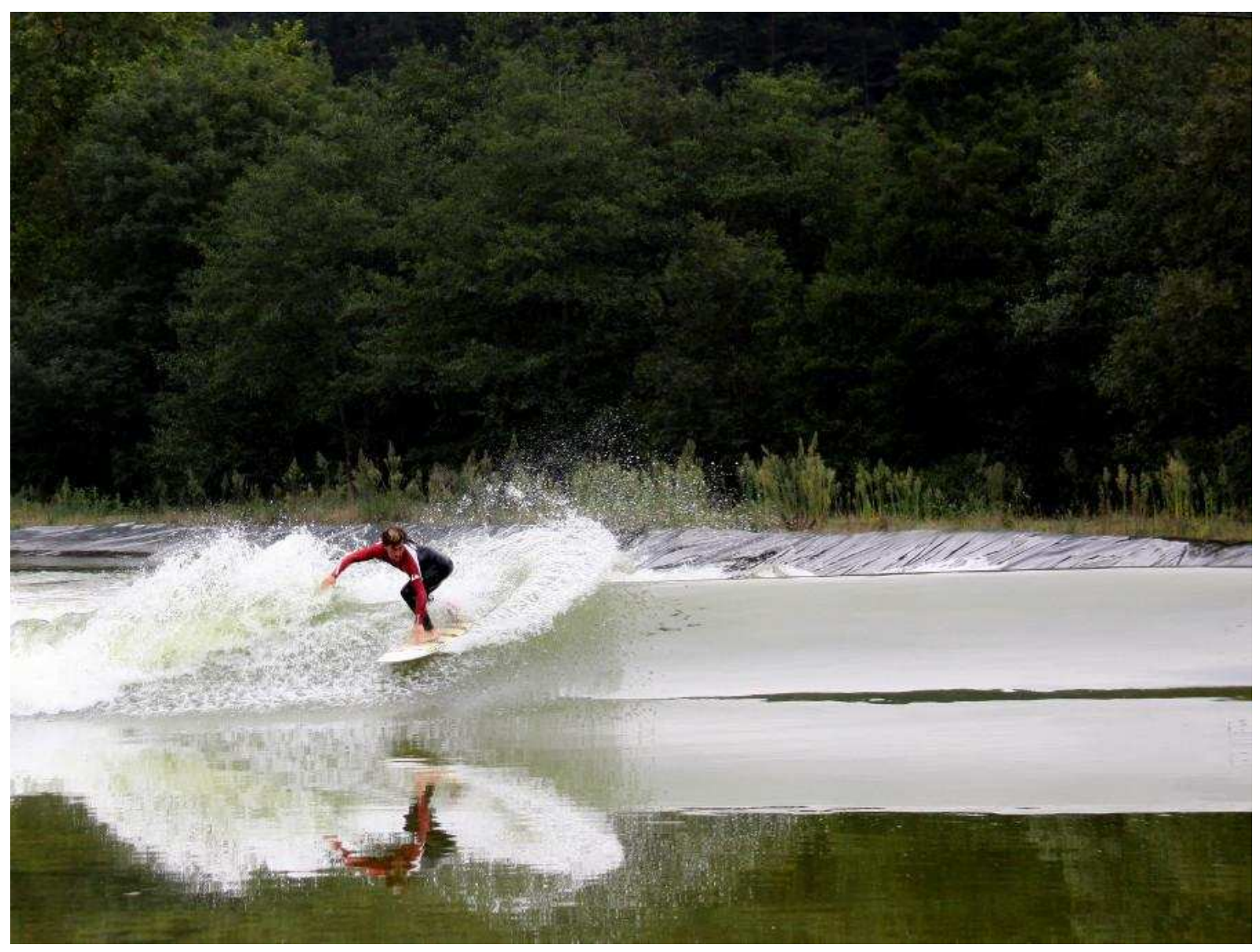

FIGURE 1. An artificial wave generated in a pool by an underwater wave making device. (C) http: //www. wavegarden. com/

wave excitation problem by moving forcing at the bottom. Similar processes are known in physics under the name of autoresonance phenomena, thoroughly studied by L. FRIEDLAND and his collaborators $[28,29,30]$.

Recently, this type of wavemakers has found an interesting application to the man-made surfing facilities [36]. The device was proven to be successful to generate high quality waves for surfing far from the Oceans. Our main goal consists in providing some elements of the modelling and theoretical analysis of this process. The second objective of this study is to provide an efficient computational procedure to determine the underwater object shape and trajectory to generate a prescribed wave profile in a given portion of the wave tank.

The problem of wave generation by moving bottom has been particularly studied in the context of tsunami waves genesis. These extreme waves are caused by sea bed displacements due to an underwater earthquake $[34,11,44,21,26]$ or a submarine landslide $[64,45$, $65,2]$. It is mainly the vertical bottom motion which contributes most to the tsunami generation by earthquakes, even if some effort has been made to take into account also for the horizontal displacement components [59, 61, 60, 43, 25]. On the other hand, the wave making mechanism studied here involves only the horizontal motion. Consequently, 
the methods and known results from the tsunami wave community cannot be directly transposed to this problem.

The wave propagation takes place in a shallow channel, so the long wave assumption can be adopted $[63,17]$. However, weak dispersive and weak nonlinear effects should be included since the resulting wave observed in experiments has some common characteristics with a solitary wave. Consequently, as the base model we choose the classical Boussinesq system derived by D.H. Peregrine (1967) [46] and generalized later by T. Wu (1987) [66], who included the time-dependent bathymetry effects. In order to simplify further the problem, we assume the wave propagation to be unidirectional and, hence, we derive a generalized forced Benjamin-Bona-Mahony (BBM) equation [3]. This equation is then discretized with a high order finite volume method [4, 23, 14, 24]. Finally, the trajectory and the shape of the underwater wavemaker are optimized in order to minimize a costfunction under some practical constraints.

From mathematical point of view our formulation can be seen as the controllability problem for the forced BBM equation [48]. Let us describe the main available results on the controllability of dispersive wave equations such as $\mathrm{KdV}[10,38]$, BBM [3] and some Boussinesq-type systems [6].

The controllability of the KdV equation

$$
u_{t}+u_{x x x}+u_{x}+u u_{x}=0, \quad x \in[0, L], t>0
$$

is well studied in the literature. The controllability and stabilization properties were obtained by L. Russell \& B.-Y. ZhANG (1993) [53] for periodic boundary conditions with an internal control. The boundary control was investigated by the same authors later [54]. The controllability of the KdV equation with Dirichlet boundary conditions was studied in the following papers [55,49,67,47, 15, 51, 12, 32, 13, 33, 40], the list of references not being exhaustive.

Let us briefly describe now some results on the controllability of the BBM (or regularized KdV) equation. Rosier \& ZHANG (2012) [52] proved the Unique Continuation Property (UCP) for the solution of BBM equation on a one dimensional torus $\mathbb{T}:=\mathbb{R} / 2 \pi \mathbb{Z}$ with small enough initial data from $H^{1}(\mathbb{T})$ with nonnegative mean values:

$$
\begin{aligned}
u_{t}-u_{t x x}+u_{x}+u u_{x}=0, & u(0)=u_{0}, \\
\int_{\mathbb{T}} u_{0}(x) \mathrm{d} x \geq 0, & \left\|u_{0}\right\|_{\infty}<3,
\end{aligned}
$$

i.e. for any open nonempty set $\omega \subset \mathbb{T}$ the only solution of (1.1) with

$$
u(x, t)=0 \text { for }(x, t) \in \omega \times(0, T)
$$

is the trivial solution $u=0$. Moreover, they proved the UCP also for BBM-type equations of the form

$$
u_{t}-u_{t x x}+[f(u)]_{x}=0,
$$

where $f \in C^{1}(\mathbb{R}), f(u) \geq 0$ for all $u \in \mathbb{R}$, and the only solution $u \in(-\delta, \delta)$ of $f(u)=0$ is $u=0$, for some number $\delta>0$. Furthermore, they consider the following control problem:

$$
u_{t}-u_{t x x}+u_{x}+u u_{x}=a(x+c t) h(x, t),
$$


where $a \in C^{\infty}$ is given and $h(x, t)$ is the control. They prove local exact controllability in $H^{s}(\mathbb{T})$ for any $s \geq 0$ and global exact controllability in $H^{s}(\mathbb{T})$ for any $s \geq 1$. A necessary and sufficient algebraic condition for approximate controllability of the BBM equation with homogeneous Dirichlet boundary conditions was given in [1]. The controllability of linearized BBM and $\mathrm{KdV}$ equations was studied in [50, 41, 69]. The controllability of a family of Boussinesq equations has been studied theoretically as well [68]. In [62], Touboul recently obtained controllability results for the heat and wave equations with a moving boundary.

The present study is organized as follows. In Section 2 we derive the governing equation. Then, this model is analysed mathematically in Section 3. The results of some numerical simulations are presented in Section 4. Finally, in Section 5 we outline the main conclusions of this study.

\section{Mathematical model}

Consider an ideal incompressible fluid of constant density in a two-dimensional domain. The horizontal independent variable is denoted by $x$ and the upward vertical one by $y$. The origin of the Cartesian coordinate system is chosen such that the line $y=0$ corresponds to the still water level. The fluid is bounded below by an impermeable bottom at $y=-h(x, t)$ and above by an impermeable free surface at $y=\eta(x, t)$. We assume that the total depth $H(x, t) \equiv h(x, t)+\eta(x, t)$ remains positive $H(x, t) \geqslant H_{0}>0$ at all times $t$. The sketch of the physical domain is shown in Figure 2. The depth-averaged horizontal velocity is denoted by $u(x, t)$ and the gravity acceleration by $g$. The fluid layer has the uniform depth $d$ everywhere, which is perturbed only by a localized object, which can move along the bottom:

$$
h(x, t)=d-\zeta(x, t), \quad \zeta(x, t)=\zeta_{0}\left(x-x_{0}(t)\right),
$$

where the function $\zeta_{0}(x)$ has a compact support and $x=x_{0}(t)$ is the trajectory of its barycenter. The meaning of the segment $\mathcal{I}=[a, b]$ is explained in Section 3.1.

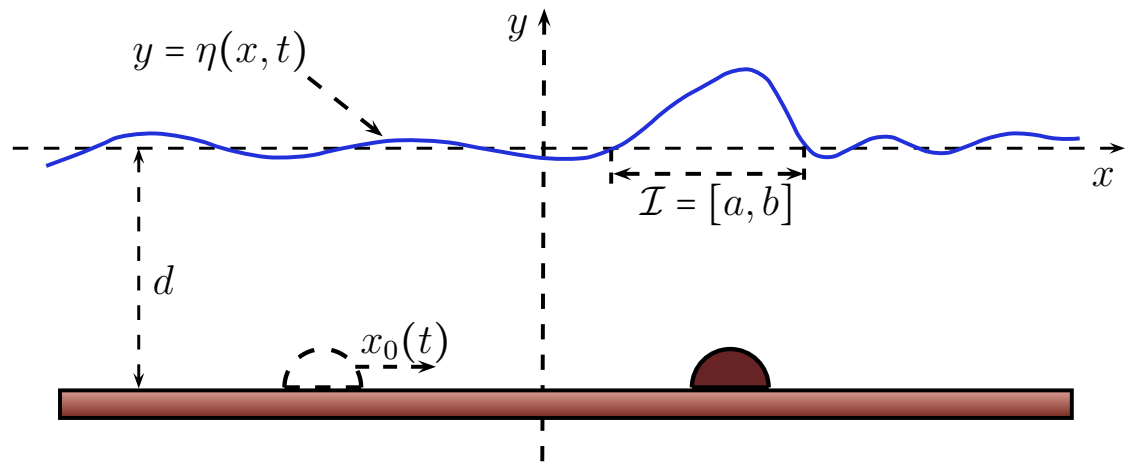

FIGURE 2. Sketch of the physical domain with an underwater object moving along the bottom. 
In 1987 T. Wu [66] derived the following Boussinesq-type system to study the generation of solitary waves by moving disturbances:

$$
\begin{aligned}
\eta_{t}+((h+\eta) u)_{x} & =-h_{t}, \\
u_{t}+u u_{x}+g \eta_{x} & =\frac{1}{2} h\left(h_{t}+(h u)_{x}\right)_{x t}-\frac{1}{6} h^{2} u_{x x t} .
\end{aligned}
$$

This system represents a further generalization of the classical Boussinesq equations derived by D.H. Peregrine (1967) [46] for the case of the moving bottom $h(x, t)$. In our work we take the system (2.2), (2.3) as the starting point. In order to simplify it further, we will switch to dimensionless variables (denoted with primes):

$$
x^{\prime}=\frac{x}{l}, \quad \eta^{\prime}=\frac{\eta}{a}, \quad h^{\prime}=\frac{h}{d}, \quad u^{\prime}=\frac{u}{\frac{g a}{\sqrt{g d}}}, \quad t^{\prime}=\frac{t}{\frac{\ell}{\sqrt{g d}}}, \text { and } \zeta^{\prime}=\frac{\zeta}{a},
$$

where $a$ and $\ell$ are the characteristic wave amplitude and wavelength correspondingly. We can compose three important dimensionless numbers which characterize the Boussinesq regime:

$$
\varepsilon:=\frac{a}{d} \ll 1, \quad \mu^{2}:=\left(\frac{d}{\ell}\right)^{2} \ll 1, \text { and } S:=\frac{\varepsilon}{\mu^{2}}=O(1),
$$

where $S$ is the so-called Stokes-Ursell number [63], which measures the relative importance of dispersive and nonlinear effects. In unscaled variables the Peregrine-Wu system takes the following form (for simplicity we drop out the primes):

$$
\begin{aligned}
\eta_{t}+((h+\varepsilon \eta) u)_{x} & =-h_{t}, \\
u_{t}+\varepsilon u u_{x}+\eta_{x} & =\frac{\mu^{2}}{2} h\left(h_{t}+(h u)_{x}\right)_{x t}-\frac{\mu^{2}}{6} h^{2} u_{x x t} .
\end{aligned}
$$

To simplify the problem, we will reduce the Boussinesq system (2.2), (2.3) to the unidirectional wave propagation. For instance, in the original work of $\mathrm{T}$. WU [66] a similar reduction to a forced $\mathrm{KdV}$ is also performed. However, the resulting model in our work will be of the BBM-type [3], since it possesses better numerical stability properties.

The reduction to the BBM equation can be done in the following way [37]. The horizontal velocity $u$ can be approximatively represented in unscaled variables as

$$
u=\eta+\varepsilon P+\mu^{2} Q+O\left(\varepsilon^{2}+\varepsilon \mu^{2}+\mu^{4}\right),
$$

where $P(x, t)$ and $Q(x, t)$ are some functions to be determined. The sign + in front of $\eta$ means that we consider the waves moving in the rightward direction. Substituting the representation (2.6) into unscaled Boussinesq equations (2.4), (2.5) yields two equivalent relations:

$$
\begin{aligned}
\eta_{t}+\eta_{x}+\varepsilon P_{x}+\mu^{2} Q_{x}+2 \varepsilon \eta \eta_{x}-\varepsilon(\zeta \eta)_{x}-\varepsilon \zeta_{t} & =O\left(\varepsilon^{2}+\varepsilon \mu^{2}+\mu^{4}\right), \\
\eta_{t}+\eta_{x}+\varepsilon P_{t}+\mu^{2} Q_{t}+\varepsilon \eta \eta_{x} & =\frac{\mu^{2}}{2} h\left(-\zeta_{t}+(h \eta)_{x}\right)_{x t}-\frac{\mu^{2}}{6} h^{2} \eta_{x x t}+O\left(\varepsilon^{2}+\varepsilon \mu^{2}+\mu^{4}\right) .
\end{aligned}
$$

By subtracting two last asymptotic relations we obtain the following compatibility condition:

$\varepsilon\left(P_{x}-P_{t}\right)+\mu^{2}\left(Q_{x}-Q_{t}\right)+\varepsilon \eta \eta_{x}-\varepsilon(\zeta \eta)_{x}=\varepsilon \zeta_{t}-\frac{\mu^{2}}{2}\left(-\zeta_{t}+\eta_{x}\right)_{x t}+\frac{\mu^{2}}{6} h^{2} \eta_{x x t}+O\left(\varepsilon^{2}+\varepsilon \mu^{2}+\mu^{4}\right)$. 
For the right-going waves we have the following identities:

$$
P_{t}=-P_{x}+O(\varepsilon), \quad Q_{t}=-Q_{x}+O(\varepsilon) .
$$

Finally, the unknown functions $P_{x}$ and $Q_{x}$ can be chosen to satisfy asymptotically the compatibility condition (2.8), which yields

$$
2 P_{x}=(\zeta \eta)_{x}-\eta \eta_{x}+\zeta_{t}, \quad 2 Q_{x}=\frac{1}{2} \zeta_{x t t}-\frac{1}{3} \eta_{x x t},
$$

where we used also the analytical representation of $h(x, t)=1-\varepsilon \zeta(x, t)$. The BBM equation in unscaled variables can be now easily obtained by substituting expressions for $P_{x}$ and $Q_{x}$ into equation (2.7):

$$
\eta_{t}+\eta_{x}+\frac{\varepsilon}{2}\left((\zeta \eta)_{x}-\eta \eta_{x}+\zeta_{t}\right)+\frac{\mu^{2}}{2}\left(\frac{1}{2} \zeta_{x t t}-\frac{1}{3} \eta_{x x t}\right)+2 \varepsilon \eta \eta_{x}-\varepsilon(\zeta \eta)_{x}=\varepsilon \zeta_{t} .
$$

Turning back to physical variables, the generalized forced BBM (gBBM) equation takes the following form:

$$
\eta_{t}+\left(\sqrt{g d} \eta+\sqrt{\frac{g}{d}}\left(\frac{3}{4} \eta^{2}-\frac{1}{2} \zeta \eta\right)\right)_{x}-\frac{d^{2}}{6} \eta_{x x t}=-\frac{1}{4} \frac{d^{2}}{\sqrt{g d}} \zeta_{x t t}+\frac{1}{2} \zeta_{t} .
$$

In subsequent sections we will use this equation to model wave-bottom interaction. However, in order to simplify the notations, we will introduce a new set of dimensionless variables, where all the lengthes are unscaled with the water depth $d$, velocities with $\sqrt{g d}$ and the time variable with $\sqrt{d / g}$. In this scaling the gBBM equation reads:

$$
\eta_{t}+\left(\eta+\frac{3}{4} \eta^{2}-\frac{1}{2} \zeta \eta\right)_{x}-\frac{1}{6} \eta_{x x t}=-\frac{1}{4} \zeta_{x t t}+\frac{1}{2} \zeta_{t}
$$

Recall that here $\eta$ is the unknown free surface elevation, and $\zeta$ is a given function, which is the topography of moving body defined by (2.1). The last equation has to be completed by appropriate initial and boundary conditions (when posed on a finite or semi-infinite domain):

$$
\eta(x, 0)=\eta_{0}(x), \quad x \in \mathbb{R} .
$$

The method that we used to get the model (2.9) is known in the literature. For instance, in [9], by the similar arguments, it was obtained the model for generation of waves by a moving boundary. The system BBM was justified also by some laboratory experiments [7].

\section{Well-posedness of the gBBM equation}

In this section we give a proof of the well-posedness of gBBM equation (2.9) in the Sobolev spaces $H^{s}:=H^{s}(\mathbb{R})$. First, we have the following result.

Theorem 1. For any $\zeta \in C^{2}\left([0, \infty), H^{s}\right) \cap C\left([0, \infty), H^{[s]+1}\right)$ and $\eta_{0} \in H^{s}, s \geq 0$ the problem (2.9), (2.10) admits a unique solution $\eta \in C\left([0, \infty), H^{s}\right)$.

Proof. Uniqueness. Let us assume that for some given functions $\zeta$ and $\eta_{0}$ our problem (2.9), (2.10) admits two different solutions $\eta_{1}$ and $\eta_{2}$. The difference $\tilde{\eta}:=\eta_{1}-\eta_{2}$ satisfies 
the following initial-value problem:

$$
\tilde{\eta}_{t}+\left(\tilde{\eta}+\frac{3}{4} \tilde{\eta}\left(\eta_{1}+\eta_{2}\right)-\frac{1}{2} \zeta \tilde{\eta}\right)_{x}-\frac{1}{6} \tilde{\eta}_{x x t}=0, \quad \tilde{\eta}(x, 0)=0 .
$$

Using Fourier transformation, we can rewrite (3.1) in the form

$$
\mathrm{i} \tilde{\eta}_{t}=\varphi\left(D_{x}\right)\left(\tilde{\eta}+\frac{3}{4} \tilde{\eta}\left(\eta_{1}+\eta_{2}\right)-\frac{1}{2} \zeta \tilde{\eta}\right)
$$

where $\varphi\left(D_{x}\right)$ is defined by $\overline{\varphi\left(D_{x}\right)} v(\xi):=\frac{\xi}{1+1 / 6 \xi^{2}} \hat{v}(\xi)$. Clearly, (3.2) implies

$$
\tilde{\eta}(x, t)=-\mathrm{i} \int_{0}^{t} \varphi\left(D_{x}\right)\left(\tilde{\eta}+\frac{3}{4} \tilde{\eta}\left(\eta_{1}+\eta_{2}\right)-\frac{1}{2} \zeta \tilde{\eta}\right) \mathrm{d} t .
$$

From the inequality

$$
\left\|\varphi\left(D_{x}\right)(u v)\right\|_{0} \leq C\|u\|_{0}\|v\|_{0}
$$

it follows that

$$
\sup _{t \in[0, T]}\|\tilde{\eta}(x, t)\|_{0} \leq C T\left(1+\left\|\eta_{1}\right\|_{0}+\left\|\eta_{2}\right\|_{0}+\|\zeta\|_{0}\right)\|\tilde{\eta}\|_{0}
$$

Thus, the application of the Gronwall inequality yields $\tilde{\eta}=0$.

Existence. For any fixed time horizon $T>0$ let us show that our problem (2.9), (2.10) has a solution $\eta \in C\left([0, T), H^{s}\right)$. J. Bona \& N. Tzvetkov (2009) [8] proved that for any given initial data $\eta_{0} \in H^{s}$ the following BBM equation

$$
u_{t}+u_{x}+u u_{x}-u_{x x t}=0, \quad u(x, 0)=u_{0}(x),
$$

admits a unique solution $u \in C\left([0, \infty), H^{s}\right)$ (for $s<0$ they proved that the system is illposed). Using a scaling argument, we have also the well-posedness of the same equation with some positive coefficients:

$$
u_{t}+\left(u+\frac{3}{4} u^{2}\right)_{x}-\frac{1}{6} u_{x x t}=0, \quad u(x, 0)=\eta_{0}(x) .
$$

We seek a solution of $(2.9),(2.10)$ in the form $\eta=u+v$, where $u \in C\left([0, \infty), H^{s}\right)$ is the solution of (3.4) and $v$ satisfies

$$
v_{t}+\left(v+\frac{3}{4}\left(v^{2}+2 u v\right)-\frac{1}{2} \zeta(v+u)\right)_{x}-\frac{1}{6} v_{x x t}=-\frac{1}{4} \zeta_{x t t}+\frac{1}{2} \zeta_{t}, \quad v(x, 0)=0 .
$$

Let us prove the existence of such $v \in C\left([0, \infty), H^{s}\right)$ by induction on $[s]$. First, we assume $[s]=0$. Taking the scalar product of $(3.5)$ with $v$ in $L^{2}$, we obtain

$$
\frac{\mathrm{d}}{\mathrm{d} t} \int_{\mathbb{R}}\left(\frac{1}{2} v^{2}(x, t)+\frac{1}{12} v_{x}^{2}(x, t)\right) \mathrm{d} x=-\int_{\mathbb{R}} \frac{1}{4} \zeta_{x t t} v \mathrm{~d} x+\int_{\mathbb{R}} \frac{1}{2} \zeta_{t} v \mathrm{~d} x+\int_{\mathbb{R}}\left(\frac{3}{2} u v-\frac{1}{2} \zeta(v+u)\right) v_{x} \mathrm{~d} x .
$$

Using the Sobolev and Hölder inequalities, we get

$$
\begin{aligned}
\frac{\mathrm{d}}{\mathrm{d} t}\|v(\cdot, t)\|_{1}^{2} \leq C\left(\left\|\zeta_{t t}(\cdot, t)\right\|_{0}\|v(\cdot, t)\|_{1}+\right. & \left\|\zeta_{t}(\cdot, t)\right\|_{0}\|v(\cdot, t)\|_{0}+\|u(\cdot, t)\|_{0}\left\|_{0}(\cdot, t)\right\|_{1}^{2} \\
& \left.+\|\zeta(\cdot, t)\|_{1}\|v(\cdot, t)\|_{1}\left(\|v(\cdot, t)\|_{0}+\|u(\cdot, t)\|_{0}\right)\right) .
\end{aligned}
$$


After integrating (3.6) on the interval $(0, t)$ we obtain

$$
\begin{array}{r}
\|v(\cdot, t)\|_{1}^{2} \leq C \sup _{t \in[0, T]}\|v(\cdot, t)\|_{1} \int_{0}^{T}\left(\left\|\zeta_{t t}(\cdot, s)\right\|_{0}+\left\|\zeta_{t}(\cdot, s)\right\|_{0}+\|u(\cdot, s)\|_{0}\|v(\cdot, s)\|_{1}+\right. \\
\left.\|\zeta(\cdot, s)\|_{1}\left(\|v(\cdot, s)\|_{0}+\|u(\cdot, s)\|_{0}\right)\right) \mathrm{d} s
\end{array}
$$

which is valid for any $t \in[0, T]$. Hence, $\sup _{t \in[0, T]}\|v(\cdot, t)\|_{1}^{2}$ also can be estimated by the right hand-side of (3.7). Dividing by $\sup _{t \in[0, T]}\|v(\cdot, t)\|_{1}^{2}$ and applying the Gronwall inequality, we deduce

$$
\begin{aligned}
& \sup _{t \in[0, T]}\|v(\cdot, t)\|_{1} \leq C \int_{0}^{T}\left(\left\|\zeta_{t t}(\cdot, s)\right\|_{0}+\left\|\zeta_{t}(\cdot, s)\right\|_{0}+\|\zeta(\cdot, s)\|_{1}\|u(\cdot, s)\|_{0}\right) \mathrm{d} s \times \\
& \exp \left(\int_{0}^{T}\left(\|u(\cdot, s)\|_{0}+\|\zeta(\cdot, s)\|_{1}\right) \mathrm{d} s\right) .
\end{aligned}
$$

Using this estimation and applying fixed point argument, we can obtain the existence of the solution in the case $s \in[0,1]$.

By induction, now we assume the existence of $v$ for $[s]<\alpha$ for some integer $\alpha>1$ and let us prove it for $[s]=\alpha$. To this end, let us take the $\frac{\mathrm{d}^{\alpha}}{\mathrm{d} x^{\alpha}}, \alpha \leq[s]$ derivative of $(3.5)$, multiply the resulting equation by $v_{\alpha}:=\frac{\mathrm{d}^{\alpha} v}{\mathrm{~d} x^{\alpha}}$ and integrating in $x$ over $\mathbb{R}$, we obtain

$$
\begin{aligned}
\frac{\mathrm{d}}{\mathrm{d} t} \int_{\mathbb{R}}\left(\frac{1}{2} v_{\alpha}^{2}(x, t)+\frac{1}{12} v_{\alpha x}^{2}(x, t)\right) \mathrm{d} x= & -\int_{\mathbb{R}}\left(\frac{1}{4}\left(\frac{\mathrm{d}^{\alpha}}{\mathrm{d} x^{\alpha}} \zeta_{x t t}\right) v_{\alpha}\right) \mathrm{d} x+\int_{\mathbb{R}}\left(\frac{1}{2}\left(\frac{\mathrm{d}^{\alpha}}{\mathrm{d} x^{\alpha}} \zeta_{t}\right) v_{\alpha}\right) \mathrm{d} x+ \\
& \int_{\mathbb{R}}\left(\frac{3}{4} \frac{\mathrm{d}^{\alpha}}{\mathrm{d} x^{\alpha}}\left(v^{2}+2 u v\right)-\frac{1}{2} \frac{\mathrm{d}^{\alpha}}{\mathrm{d} x^{\alpha}}(\zeta(v+u))\right) v_{\alpha x} \mathrm{~d} x .
\end{aligned}
$$

All the terms can be treated as above, except the term $\int_{\mathbb{R}} \frac{\mathrm{d}^{\alpha}}{\mathrm{d} x^{\alpha}}\left(v^{2}\right) v_{\alpha x} \mathrm{~d} x$, which is not zero in general. Using the induction hypothesis and the fact that $\alpha-1 \geq 1$, we can estimate

$$
\left|\int_{\mathbb{R}} \frac{\mathrm{d}^{\alpha}}{\mathrm{d} x^{\alpha}}\left(v^{2}\right) v_{\alpha x} \mathrm{~d} x\right| \leq C\left\|v_{\alpha}\right\|_{1}^{2}\|v\|_{\alpha-1}<M\left\|v_{\alpha}\right\|_{1}^{2} .
$$

Using the last estimation along with (3.8), the Sobolev and Hölder inequalities, as above, we prove the required estimation for $v_{\alpha}$, which completes the proof.

\subsection{Optimization problem}

In this section we turn to the optimization problem for the gBBM equation (2.9). We assume that the wave making piston is a solid, non-deformable object. Thus, its shape, given by a localized function $\zeta_{0}(x)$, is preserved during the motion and it is sufficient to prescribe the trajectory of its barycenter only $x=x_{0}(t)$. Consequently, the time-dependent 
bathymetry is given by the following equation

$$
h(x, t)=d-\zeta_{0}\left(x-x_{0}(t)\right) .
$$

The piston shape $\zeta_{0}(x)$ and its trajectory $x_{0}(t)$ will be determined as a solution of the optimization problem. More precisely, in the next section we will find numerically these functions in order to produce the largest possible wave (in $L_{2}$ sense) in a given subinterval $\mathcal{I}=[a, b]$ of the numerical wave tank at some fixed time $T>0$. In other words, we minimize the following functional:

$$
J\left(x_{0}, \zeta_{0}\right)=-\int_{\mathcal{I}} \eta(x, T)^{2} \mathrm{~d} x \longrightarrow \min ,
$$

where $\eta(x, t)$ is the solution of $(2.9),(2.10)$. The existence of this solution is proven in the following

Theorem 2. For any constants $\varepsilon, M>0$, there exists $\left(x_{0}^{*}, \zeta_{0}^{*}\right) \in B_{M}$ such that

$$
J\left(x_{0}^{*}, \zeta_{0}^{*}\right)=\inf _{\left(x_{0}, \zeta_{0}\right) \in B_{M}} J\left(x_{0}, \zeta_{0}\right),
$$

where $B_{M}$ is a closed ball in $H^{2+\varepsilon}[0, T] \times H_{0}^{2+\varepsilon}([0,1])$ centered at origin with radius $M$.

Proof. Let $\left(x_{0}^{n}, \zeta_{0}^{n}\right)$ be an arbitrary minimizing sequence of $J$. Since $H^{2+\varepsilon}[0, T] \times H_{0}^{2+\varepsilon}([0,1])$ is Hilbert space, extracting a subsequence, if it is necessary, we can assume that there is $\left(x_{0}^{*}, \zeta_{0}^{*}\right) \in B_{M}$ such that $\left(x_{0}^{n}, \zeta_{0}^{n}\right) \rightarrow\left(x_{0}^{*}, \zeta_{0}^{*}\right)$ in $B_{M}$.

Let us denote $\eta^{n}$ the solution of $(2.9),(2.10)$ with $\zeta=\zeta^{n}:=\zeta_{0}^{n}\left(x-x_{0}^{n}(t)\right)$. Let us show that we have $\eta^{n}(T) \rightarrow \eta^{*}(T)$ in $L^{2}$, where $\eta^{*}$ is the solution of $(2.9)$, (2.10) with $\zeta=\zeta^{*}$. Indeed, for $\tilde{\eta}^{n, m}:=\eta^{n}-\eta^{m}$ we have

$\tilde{\eta}_{t}^{n, m}+\left(\tilde{\eta}^{n, m}+\frac{3}{4} \tilde{\eta}^{n, m}\left(\eta^{n}+\eta^{m}\right)-\frac{1}{2} \zeta^{n} \tilde{\eta}^{n, m}-\frac{1}{2} \zeta^{n, m} \tilde{\eta}^{m}\right)_{x}-\frac{1}{6} \tilde{\eta}_{x x t}^{n, m}=-\frac{1}{4} \tilde{\zeta}_{x t t}^{n, m}+\frac{1}{2} \tilde{\zeta}_{t}^{n, m} . \quad \tilde{\eta}^{n, m}(x, 0)=0$.

Since $\zeta_{t t}^{n} \rightarrow \zeta_{t t}^{*}:=\partial_{t t}\left(\zeta_{0}^{*}\left(x-x_{0}^{*}(t)\right)\right)$ in $L^{2}\left([0, T], L^{2}\right)$, multiplying $(3.10)$ in $L^{2}$ by $\tilde{\eta}^{n, m}$, integrating by parts and applying the Gronwall inequality, we obtain that $\eta^{n}$ is a Cauchy sequence in $H^{1}$. Hence,

$$
J\left(x_{0}^{*}, \zeta_{0}^{*}\right)=\lim _{n \rightarrow \infty} J\left(x_{0}^{n}, \zeta_{0}^{n}\right) .
$$

This completes the proof of the theorem.

However, in practice, the functional (3.9) has to be completed by appropriate constraints in order to provide a solution realizable in practice. For example, the speed of the underwater piston is limited by technological and energy consumption limitations. Some more realistic formulations will be addressed numerically in the next Section.

\section{Numerical results}

In order to discretize the gBBM equation (2.9), posed on a finite interval $[\alpha, \beta]$, we use a modern high-order finite volume scheme with the FVCF flux [31] and the UNO2 reconstruction [35]. The combination of these numerical ingredients has been extensively tested 


\begin{tabular}{lr}
\hline \hline Parameter & Value \\
\hline \hline Computational domain $[\alpha, \beta]:$ & {$[-5,10] \mathrm{m}$} \\
Wave quality evaluation area $[a, b]:$ & {$[0,6] \mathrm{m}$} \\
Number of discretization points $N:$ & 1000 \\
CFL number: & 1.95 \\
Gravity acceleration $g$ : & $9.8 \mathrm{~m} \mathrm{~s}^{-2}$ \\
Undisturbed water depth $d:$ & $1.0 \mathrm{~m}$ \\
Final simulation time $T:$ & $6.0 \mathrm{~s}$ \\
Piston motion total time $T_{f}:$ & $4.0 \mathrm{~s}$ \\
Piston length $\ell_{0}$ : & $1.0 \mathrm{~m}$ \\
Piston maximal height $a_{0} / d:$ & 0.12 \\
Piston starting point $x_{0}^{0}:$ & $0.0 \mathrm{~m}$ \\
Upper bound of the piston position $x_{\max }:$ & $4.5 \mathrm{~m}$ \\
Upper bound of the piston speed $v_{f}:$ & $1.5 \mathrm{~m} / \mathrm{s}$ \\
Wave generation limit $x_{f}:$ & $1.0 \mathrm{~m}$ \\
$\mathcal{N}$-wave solution ceter $x_{m}$ : & $2.0 \mathrm{~m}$ \\
\hline \hline
\end{tabular}

TABLE 1. Values of various parameters used in numerical computations.

and validated in the context of the unidirectional wave models [24] and Boussinesq-type equations $[22,23]$. For the time-discretization, we use the third-order Runge-Kutta scheme, which is also used in the ode23 function in Matlab [56]. In all experiments presented below we assume that the water layer is initially at rest:

$$
\eta(x, 0)=\eta_{0}(x) \equiv 0 .
$$

The computational domain $[\alpha, \beta]$ is discretized in $N$ equal subintervals, called usually the control volumes. The time step is chosen locally in order to satisfy the following CFL condition [16] used in shallow-water models:

$$
\Delta t \leq \frac{\Delta x}{\max _{1 \leq i \leq N} u_{i}+\sqrt{g d}} .
$$

The values of all physical and numerical parameters used in simulations are given in Table 1.

On the left and right boundaries we apply the Neumann-type boundary conditions which do not produce reflections. In any case, we stop the simulation before the generated wave reaches the right boundary. We recall that the gBBM equations (2.9) describes the unidirectional (rightwards, for instance) wave propagation. So, the influence of the left boundary condition is negligible.

Let us describe the constraints that we impose on the shape $\zeta_{0}(x) \geq 0$ and trajectory $x_{0}(t)$ of the underwater wavemaker. First of all, we fix the length $2 \ell_{0}$ of this object. Then, we assume that its height is also bounded:

$$
\max _{x \in \mathbb{R}} \frac{\zeta_{0}(x)}{d} \leq a_{0}
$$


We allow the piston to move during the first $T_{f} \mathrm{~s}$, its motion always starts at the same initial point $x_{0}^{0}$ and it is confined to some wave generation area $\left[x_{0}(0), x_{f}\right] \subseteq[\alpha, \beta]$ :

$$
\operatorname{supp} x_{0}^{\prime}(t) \subseteq\left[0, T_{f}\right], \quad x_{0}(0)=x_{0}^{0}, \quad x_{0}^{0} \leq x_{0}(t) \leq x_{f}, \quad \forall t \in\left[0, T_{f}\right] .
$$

However, the cost function $J\left(x_{0}, \zeta_{0}\right)$ is evaluated at time $T>T_{f}$ so that the generated waveform can evolve further into the desired shape.

Moreover, we require that the piston speed and acceleration are bounded, since too fast motions are difficult to realize in practice because of the gradually increasing energy consumption:

$$
\sup _{t \in\left[0, T_{f}\right]}\left(\left|x_{0}^{\prime}(t)\right|+\sqrt{\frac{d}{g}}\left|x_{0}^{\prime \prime}(t)\right|\right) \leq v_{f}
$$

In order to parametrize the wavemaker shape, we use only three degrees of freedom $\zeta_{0}\left(-\frac{\ell_{0}}{2}\right), \zeta_{0}(0), \zeta_{0}\left(\frac{\ell_{0}}{2}\right)$ which represent the height of the object in three points equally spaced on the $\operatorname{supp} \zeta_{0}$. Finally, the continuous shape is reconstructed by applying the interpolation with cubic splines ${ }^{1}$ through the following points:

$$
\left(-\ell_{0}, 0\right), \quad\left(-\frac{\ell_{0}}{2}, \zeta_{0}\left(-\frac{\ell_{0}}{2}\right)\right), \quad\left(0, \zeta_{0}(0)\right), \quad\left(\frac{\ell_{0}}{2}, \zeta_{0}\left(\frac{\ell_{0}}{2}\right)\right), \quad\left(\ell_{0}, 0\right)
$$

In a similar way we proceed with the parametrization of the piston trajectory $x_{0}(t)$ which is represented with 4 degrees of freedom (three in the interior of the interval $\left(0, T_{f}\right)$ and the final point $x_{0}\left(T_{f}\right)$ which is not fixed as in the case of $\left.\zeta_{0}\right)$. Obviously, more degrees of freedom can be taken into account when it is needed for a specific application. However, the number of degrees of freedom determines the dimension of the phase space where we seek for the optimal solution. In examples below we operate in a closed subset of $\mathbb{R}^{7}$. In order to obtain an approximate solution to our constrained optimization problem, we use the function fmincon of the Matlab Optimization Toolbox. This solver is a gradient-based optimization procedure which uses the SQP algorithm. The iterative process is stopped when the default tolerances are met or the maximal number of iterations is reached. In all simulations presented below the convergence of the algorithm has been achieved.

As the first numerical example, we minimize the functional $J\left(x_{0}, \zeta_{0}\right)$ subject to constraints described above. Basically, this cost function measures the wave deviation from the still water level in a fixed portion $[a, b]$ of the wave tank. consequently, bigger waves in this interval will provide lower values to the functional $J$. The result of the numerical optimisation procedure is represented on Figure 3. The free surface elevation computed at the final time $T$ is shown on Figure 3(a). One can see that in the region of interest $[a, b]=[2,4] \mathrm{m}$ we have a big depression wave which is followed by a wave of elevation. We make a conclusion that we succeeded to generate a wave suitable for surfing purposes in artificial environments. The computed shape of the underwater object is shown on Figure 3(b) and its trajectory is represented on Figure 3(c). It is interesting to note that the computed optimal shape is composed of two bumps. The piston trajectory can be conditionally decomposed into three parts. During the first $1.25 \mathrm{~s}$ we have a stage of slow motion, which is followed by a rapid acceleration and, during the last $0.75 \mathrm{~s}$, we can observe a backward

\footnotetext{
${ }^{1}$ Cubic splines ensure that the interpolant belongs to the class $C^{2}$.
} 


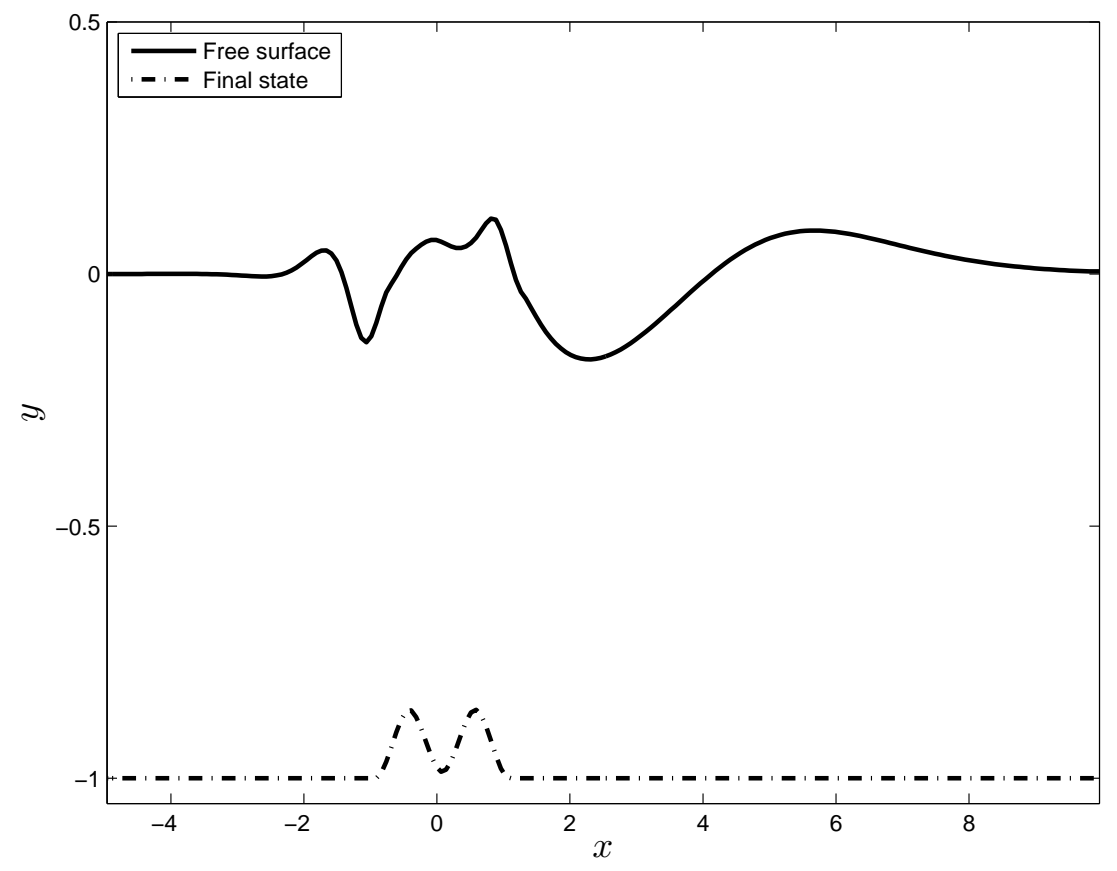

(a) Free surface elevation

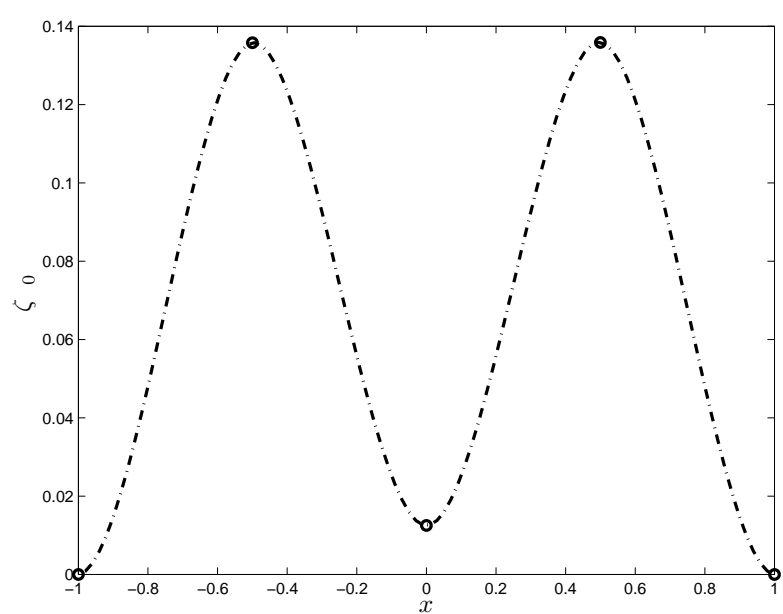

(b) Piston shape

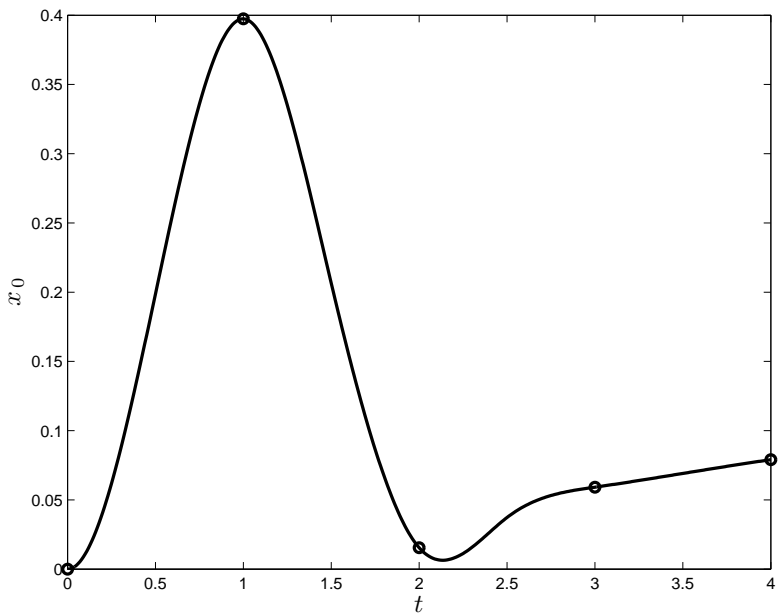

(c) Piston trajectory

FiguRE 3. Computed numerically the optimal piston shape and its trajectory which minimize the functional $J\left(x_{0}, \zeta_{0}\right)$.

motion of the piston before it is frozen in its final point. The wave has $T-T_{f}=4 \mathrm{~s}$ to evolve before its quality is estimated according the functional $J\left(x_{0}, \zeta_{0}\right)$.

Since the choice of the functional to minimize is far from being unique, we decided to perform some additional tests. Instead of maximizing the wave height, one can try to maximize, for example, the wave steepness in a given portion of the wave tank. In other 
words, we will minimize the following functional (subject to the same constraints as above):

$$
J_{1}\left(x_{0}, \zeta_{0}\right)=-\int_{\mathcal{I}} \eta_{x}(x, T) \mathrm{d} x .
$$

The result of the numerical optimization procedure is shown on Figure 4. One can see on the free surface snapshot 4(a) that effectively the wave became steeper. The optimal shape of the wavemaker is almost the same as for the functional $J\left(x_{0}, \zeta_{0}\right)$. However, the piston trajectory is almost monotonic and close to the uniform motion. This solution might be easier to realize in practice.

We can also simply minimize the mismatch between the obtained solution and a fixed desired wave profile:

$$
J_{2}\left(x_{0}, \zeta_{0}\right)=\int_{\mathcal{I}}\left(\eta(x, T)-\eta_{T}(x)\right)^{2} \mathrm{~d} x
$$

where $\eta_{T}(x)$ is a given function on the interval $\mathcal{I}$.

To illustrate this concept, in numerical computations we take the $\mathcal{N}$-wave ansatz put forward by S. TAdepalli \& C. Synolakis $(1994,1996)[57,58]$ :

$$
\eta_{T}^{(1)}(x)=\left(x-x_{m}\right) \operatorname{sech}^{2}\left(x-x_{m}\right), \quad \eta_{T}^{(2)}(x)=-\left(x-x_{m}\right) \operatorname{sech}^{2}\left(x-x_{m}\right) .
$$

The first profile $\eta_{T}^{(1)}(x)$ corresponds to the leading elevation $\mathcal{N}$-wave solution (LEN), while the second function $\eta_{T}^{(2)}(x)$ is a typical leading depression $\mathcal{N}$-wave (LDN). The results of optimization procedures are shown on Figures 5 and 6 . One can notice that the resulting optimal shapes of the wavemaker are completely different (see Figures 5(b) and 6(b)). For the surfing applications the LDN wave might be more interesting. It requires also more uniform piston motion comparing to the LEN wave (see Figures 5(c) and 6(c)).

In the final experiment are the target state is the solitary wave for gBBM:

$$
\eta_{T}^{(3)}(x)=2(c-1) \operatorname{sech}^{2}\left(\frac{\sqrt{1-c^{-1}}}{2}\left|x-x_{m}\right|\right) .
$$

As one can notice we can find the shapes of the wavemaker which generates waves close to the solitary waves for gBBM see Figures 7).

Remark 1. The arguments used to prove Theorem 2 can be also applied to show the existence of minimizers for the functionals $J_{1}\left(x_{0}, \zeta_{0}\right)$ and $J_{2}\left(x_{0}, \zeta_{0}\right)$. 


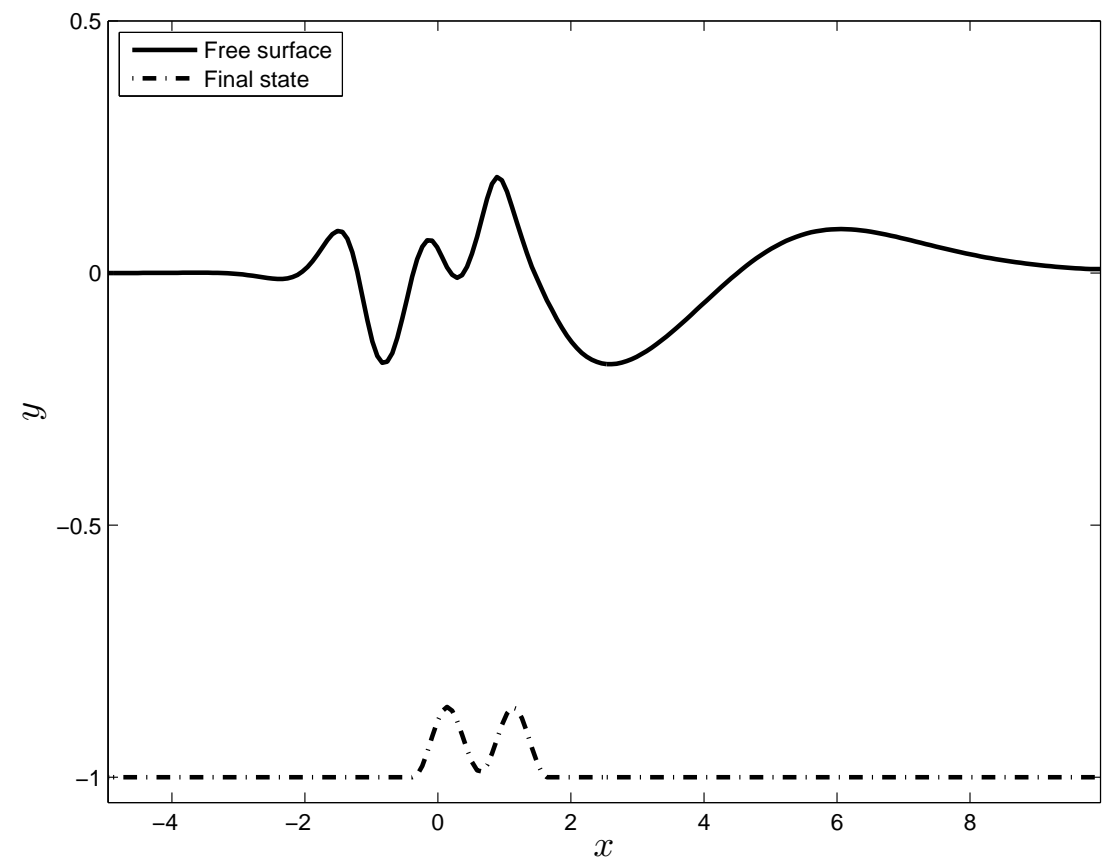

(a) Free surface elevation

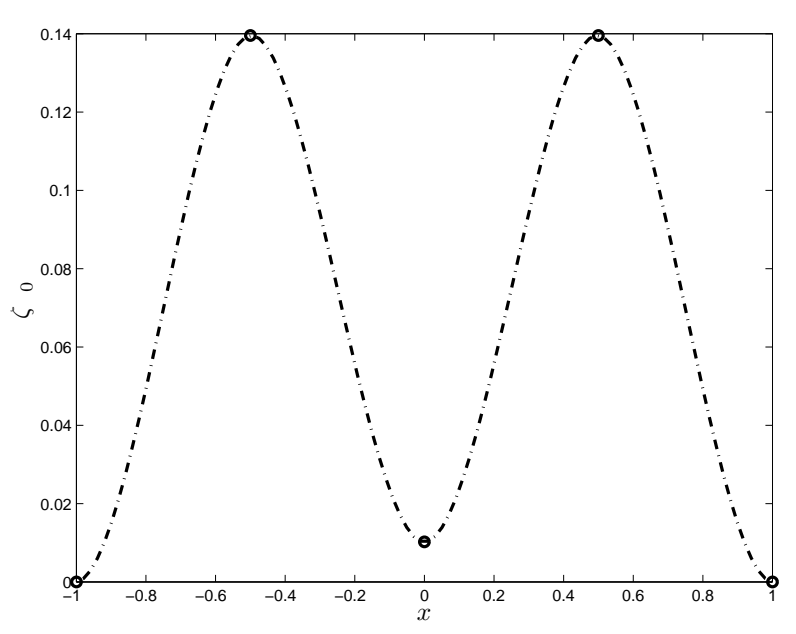

(b) Piston shape

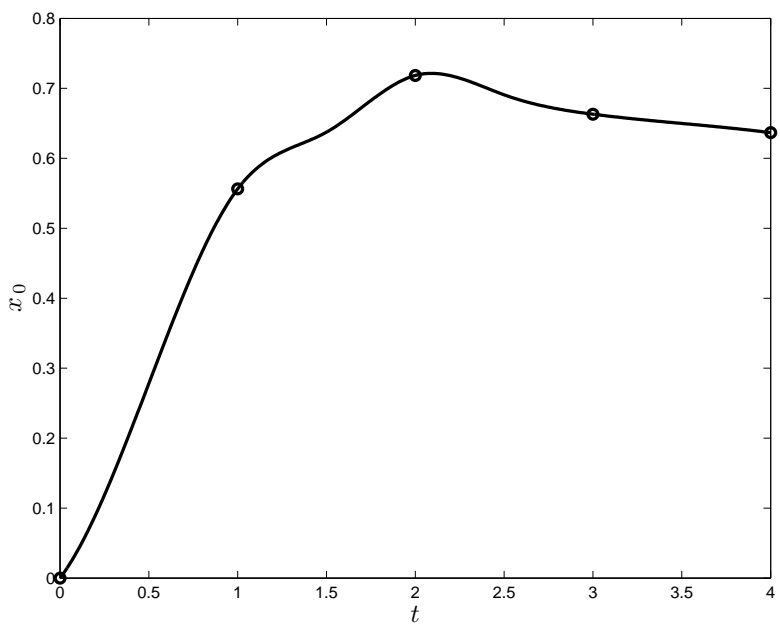

(c) Piston trajectory

FiguRE 4. Computed numerically the optimal piston shape and its trajectory which minimize the functional $J_{1}\left(x_{0}, \zeta_{0}\right)$. 


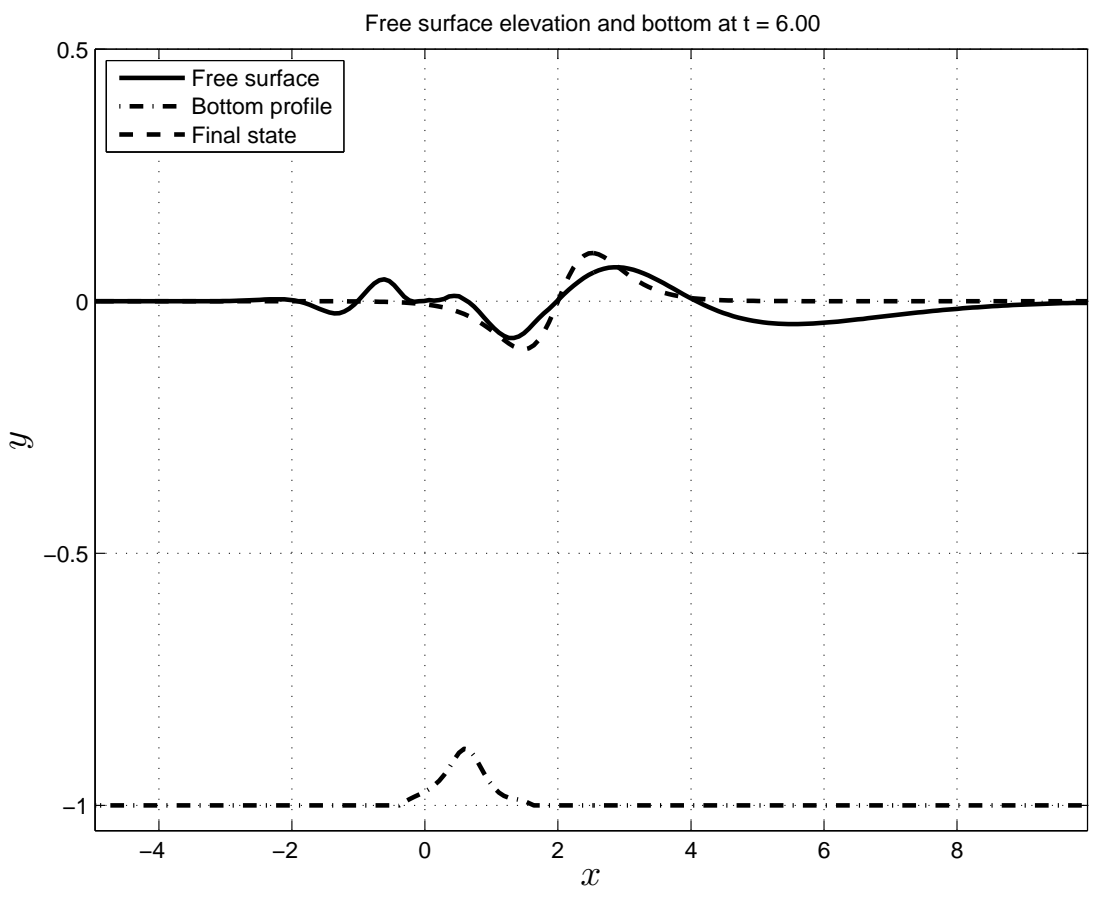

(a) Free surface elevation

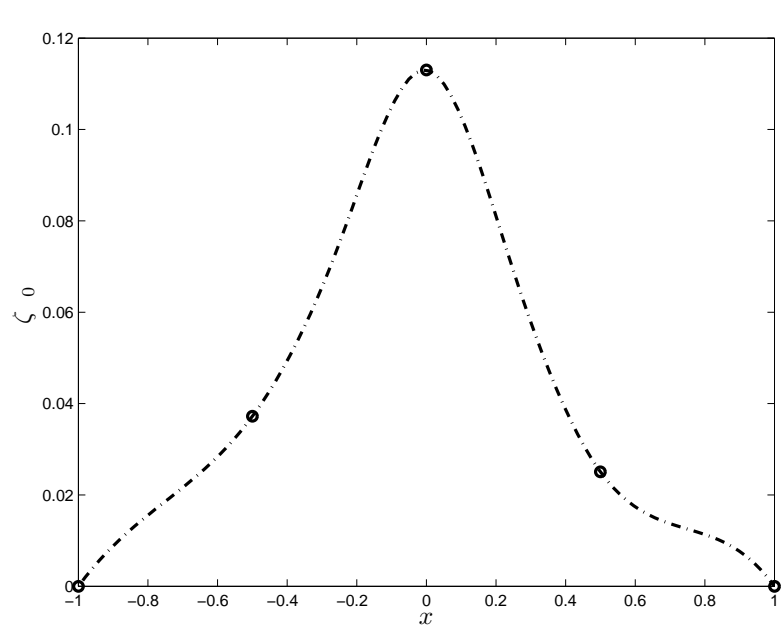

(b) Piston shape

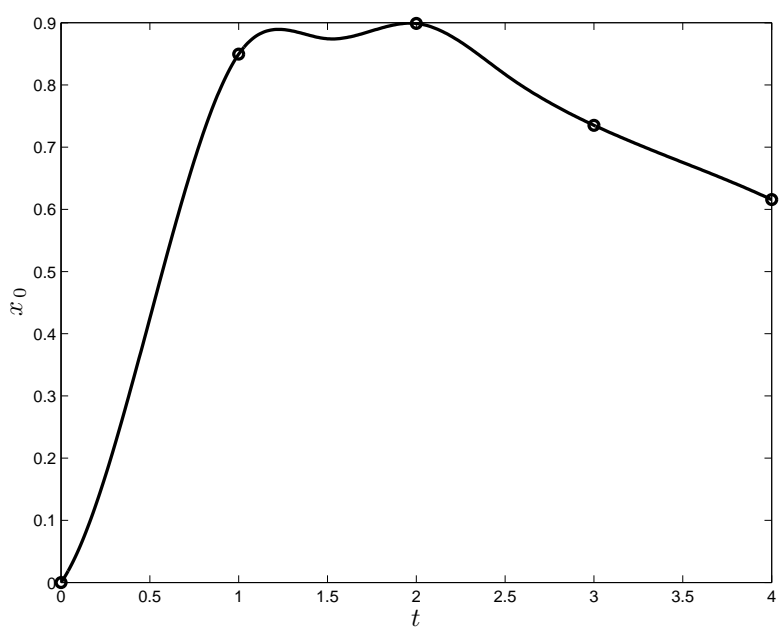

(c) Piston trajectory

FiguRE 5. Computed numerically the optimal piston shape and its trajectory which minimize the functional $J_{2}\left(x_{0}, \zeta_{0}\right)$ and the terminal state $\eta_{T}^{(1)}(x)=(x-$ $\left.x_{m}\right) \operatorname{sech}^{2}\left(x-x_{m}\right)$. 


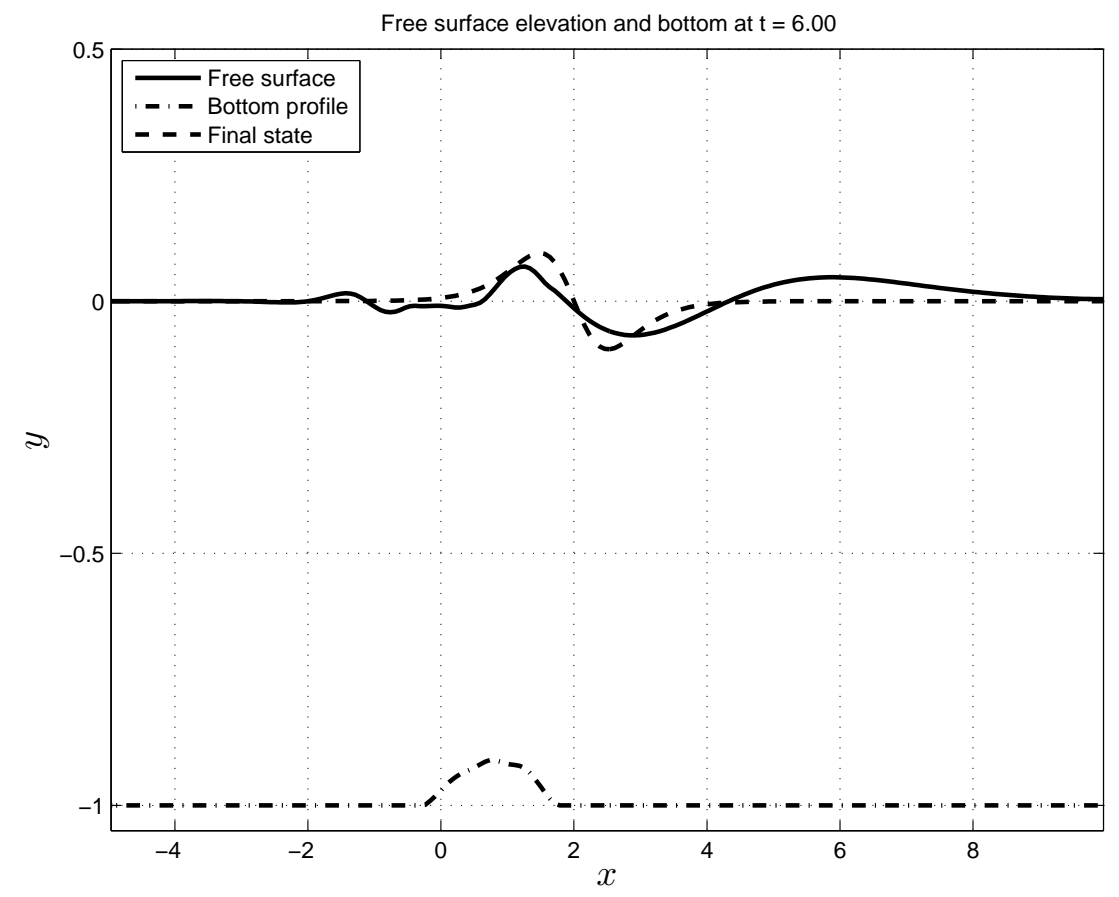

(a) Free surface elevation

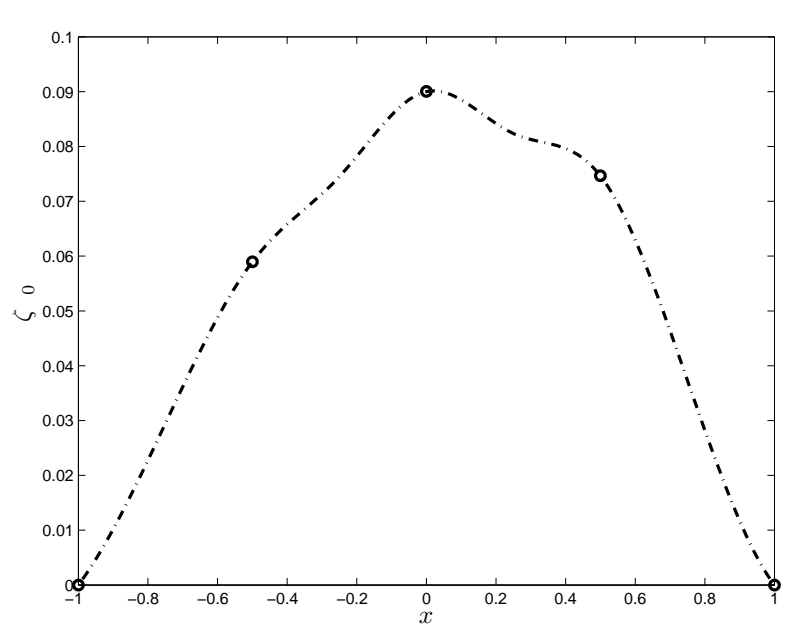

(b) Piston shape

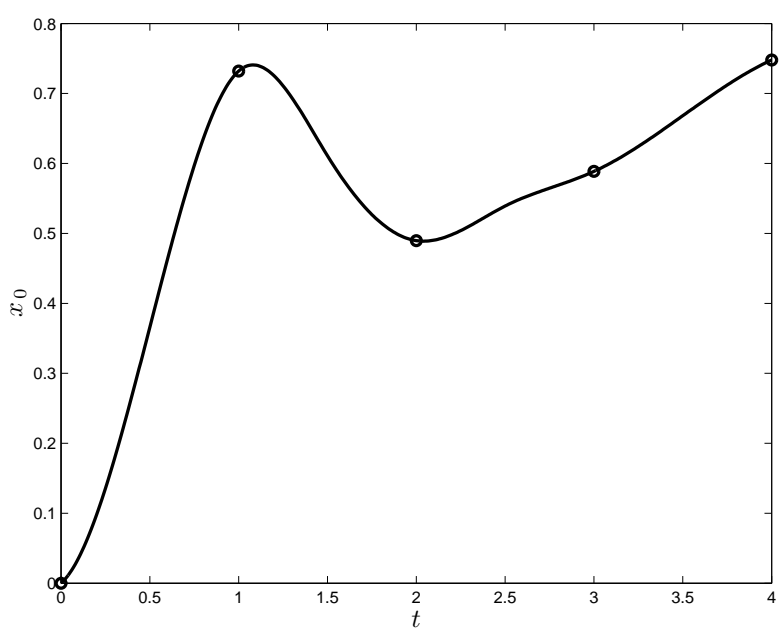

(c) Piston trajectory

FIGURE 6. Computed numerically the optimal piston shape and its trajectory which minimize the functional $J_{2}\left(x_{0}, \zeta_{0}\right)$ and the terminal state $\eta_{T}^{(1)}(x)=-(x-$ $\left.x_{m}\right) \operatorname{sech}^{2}\left(x-x_{m}\right)$. 


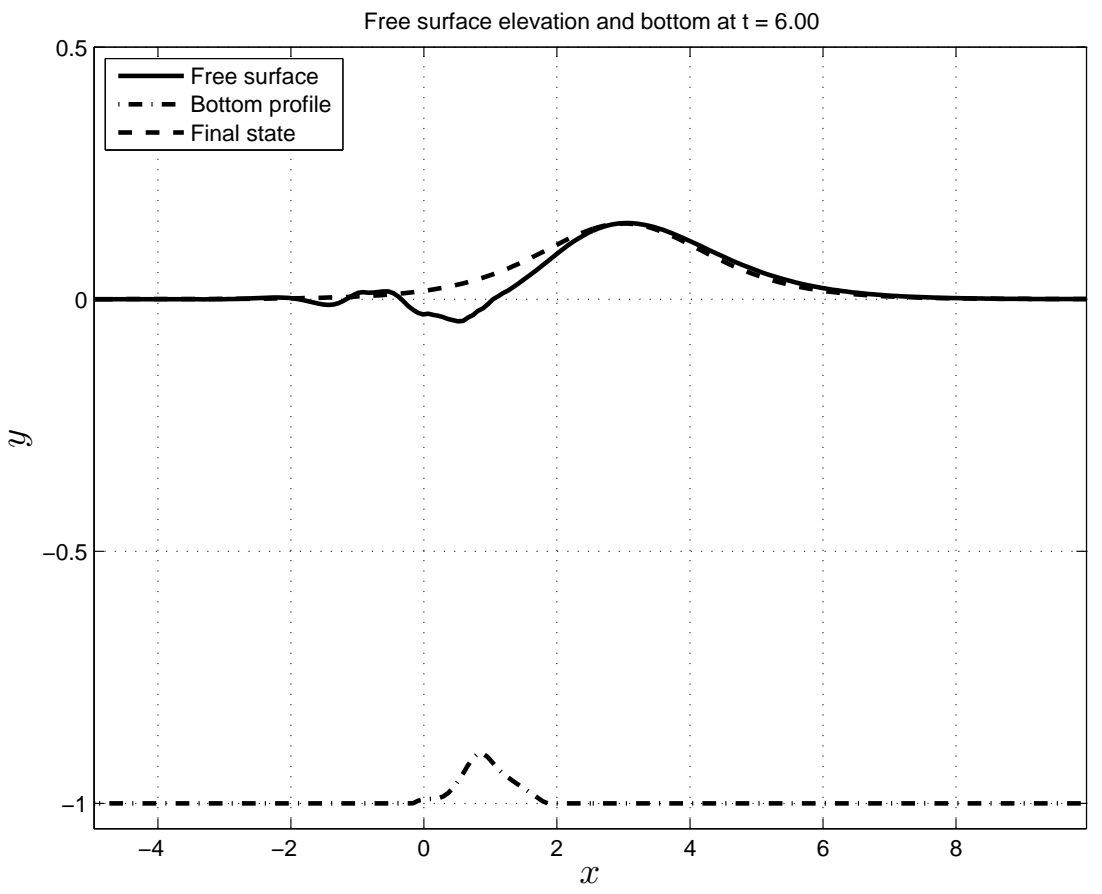

(a) Free surface elevation

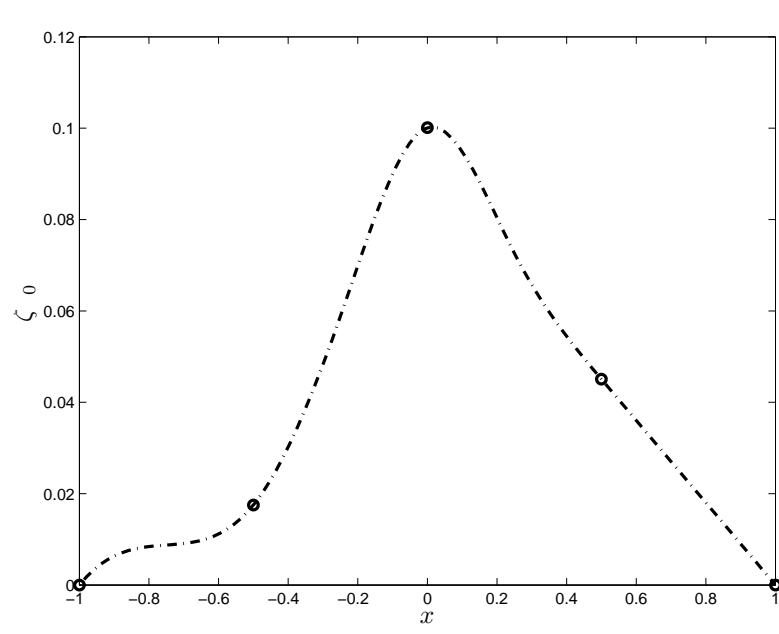

(b) Piston shape

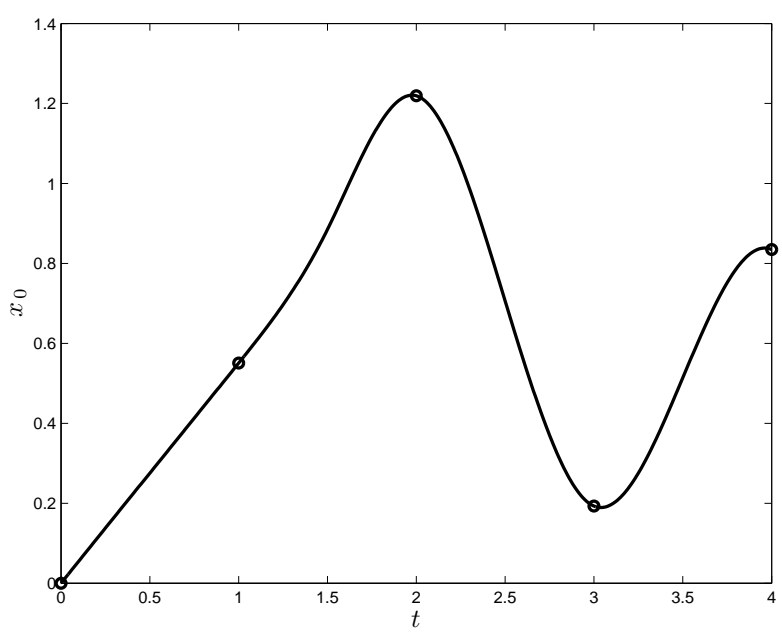

(c) Piston trajectory

FIGURE 7. Computed numerically the optimal piston shape and its trajectory which minimize the functional $J_{2}\left(x_{0}, \zeta_{0}\right)$ and the terminal state $\eta_{T}^{(2)}(x)=(c-$ 1) $\operatorname{sech}^{2}\left(\frac{\sqrt{1-c^{-1}}}{2}\left|x-x_{m}\right|\right)$. 


\section{Conclusions}

In the present work we considered the water wave generation problem by disturbances moving along the bottom. This problem has many important applications going even to the design of artificial surfing facilities [36]. In order to study the formation of water waves due to the motion of the underwater piston, we derived a generalized forced BBM (gBBM) equation. The existence and uniqueness of its solutions were rigorously established. The trajectory of the piston is determined as the solution of a thoroughly formulated optimization problem. The existence of minimizers is also proven. Finally, the theoretical developments of this study are illustrated with numerical examples where we solve several constrained optimization problems with various forms of the cost functional. The resulting solutions are compared and discussed.

In future studies this problem will be addressed in the context of more complete bidirectional wave propagation models of Boussinesq-type $[5,19,42,18]$. The optimization algorithm can be also further improved by evaluating the gradients analytically, for example. From physical point of view, one may want to include some weak dissipative effects for more realistic wave description [20].

\section{Acknowledgements}

H. NeRsisyan and E. ZuAzUA were supported by the project ERC - AdG FP7-246775 NUMERIWAVES, the Grant PI2010-04 of the Basque Government, the ESF Research Networking Program OPTPDE and Grant MTM2011-29306 of the MINECO, Spain. D. DUTYKH acknowledges the support from ERC under the research project ERC-2011-AdG 290562-MULTIWAVE. Also he would like to acknowledge the hospitality and support of the Basque Center for Applied Mathematics (BCAM) during his visits.

\section{References}

[1] N. Adames, H. Leiva, and J. Sanchez. Controllability of the Benjamin-Bona-Mahony Equation. Divulgaciones Matematicas, 16(1):29-37, 2008. 3

[2] S. A. Beisel, L. B. Chubarov, and G. S. Khakimzyanov. Simulation of surface waves generated by an underwater landslide moving over an uneven slope. Russ. J. Numer. Anal. Math. Modelling, 26(1):1738, 2011. 2

[3] T. B. Benjamin, J. L. Bona, and J. J. Mahony. Model equations for long waves in nonlinear dispersive systems. Philos. Trans. Royal Soc. London Ser. A, 272:47-78, 1972. 3, 5

[4] F. Benkhaldoun and M. Seaid. New finite-volume relaxation methods for the third-order differential equations. Commun. Comput. Phys., 4:820-837, 2008. 3

[5] J. L. Bona and M. Chen. A Boussinesq system for two-way propagation of nonlinear dispersive waves. Physica D, 116:191-224, 1998. 18

[6] J. L. Bona, M. Chen, and J.-C. Saut. Boussinesq equations and other systems for small-amplitude long waves in nonlinear dispersive media: II. The nonlinear theory. Nonlinearity, 17:925-952, 2004. 3

[7] J. L. Bona, W. G. Pritchard, and L. R. Scott. An Evaluation of a Model Equation for Water Waves. Phil. Trans. R. Soc. Lond. A, 302:457-510, 1981. 6

[8] J. L. Bona and N. Tzvetkov. Sharp well-posedness results for the BBM equation. Discrete Contin. Dyn. Syst., 23:1241-1252, 2009. 7 
[9] J. L. Bona and V. Varlamov. Wave generation by a moving boundary. Nonlinear partial differential equations and related analysis, 371:41-71, 2005. 6

[10] J. Boussinesq. Essai sur la theorie des eaux courantes. Mémoires présentés par divers savants à l'Acad. des Sci. Inst. Nat. France, XXIII:1-680, 1877. 3

[11] R. D. Braddock, P. van den Driessche, and G. W. Peady. Tsunamis generation. J. Fluid Mech., 59(4):817-828, 1973. 2

[12] E. Cerpa. Exact controllability of a nonlinear Korteweg-de Vries equation on a critical spatial domain. SIAM Journal on Control and Optimization, 46:877-899, 2007. 3

[13] E. Cerpa and E. Crepeau. Boundary controllability for the nonlinear Korteweg-de Vries equation on any critical domain. Ann. I. H. Poincaré, 26:457-475, 2009. 3

[14] F. Chazel, D. Lannes, and F. Marche. Numerical simulation of strongly nonlinear and dispersive waves using a Green-Naghdi model. J. Sci. Comput., 48:105-116, 2011. 3

[15] J.-M. Coron and E. Crepeau. Exact boundary controllability of a nonlinear KdV equation with a critical length. J. Eur. Math. Soc., 6:367-398, 2004. 3

[16] R. Courant, K. Friedrichs, and H. Lewy. Über die partiellen Differenzengleichungen der mathematischen Physik. Mathematische Annalen, 100(1):32-74, 1928. 10

[17] W. Craig and M. D. Groves. Hamiltonian long-wave approximations to the water-wave problem. Wave Motion, 19:367-389, 1994. 3

[18] V. A. Dougalis and D. E. Mitsotakis. Theory and numerical analysis of Boussinesq systems: A review. In N. A. Kampanis, V. A. Dougalis, and J. A. Ekaterinaris, editors, Effective Computational Methods in Wave Propagation, pages 63-110. CRC Press, 2008. 18

[19] V. A. Dougalis, D. E. Mitsotakis, and J.-C. Saut. On initial-boundary value problems for a Boussinesq system of BBM-BBM type in a plane domain. Discrete Contin. Dyn. Syst., 23(4):1191-1204, 2009. 18

[20] D. Dutykh and F. Dias. Dissipative Boussinesq equations. C. R. Mecanique, 335:559-583, 2007.18

[21] D. Dutykh and F. Dias. Tsunami generation by dynamic displacement of sea bed due to dip-slip faulting. Mathematics and Computers in Simulation, 80(4):837-848, 2009. 2

[22] D. Dutykh, T. Katsaounis, and D. Mitsotakis. Dispersive wave runup on non-uniform shores. In J. et al. Fort, editor, Finite Volumes for Complex Applications VI - Problems \& Perspectives, pages 389-397, Prague, 2011. Springer Berlin Heidelberg. 9

[23] D. Dutykh, T. Katsaounis, and D. Mitsotakis. Finite volume schemes for dispersive wave propagation and runup. J. Comput. Phys, 230(8):3035-3061, Apr. 2011. 3, 9

[24] D. Dutykh, T. Katsaounis, and D. Mitsotakis. Finite volume methods for unidirectional dispersive wave models. Int. J. Num. Meth. Fluids, 71:717-736, 2013. 3, 9

[25] D. Dutykh, D. Mitsotakis, L. B. Chubarov, and Y. I. Shokin. On the contribution of the horizontal sea-bed displacements into the tsunami generation process. Ocean Modelling, 56:43-56, July 2012.2

[26] D. Dutykh, D. Mitsotakis, X. Gardeil, and F. Dias. On the use of the finite fault solution for tsunami generation problems. Theor. Comput. Fluid Dyn., 27:177-199, Mar. 2013. 2

[27] J. E. Feir. Discussion: Some Results From Wave Pulse Experiments. Proceedings of the Royal Society of London. Series A, Mathematical and Physical Sciences, 299(1456):54-58, 1967. 1

[28] L. Friedland. Autoresonance of coupled nonlinear waves. Physical Review E, 57(3):3494-3501, Mar. 1998. 2

[29] L. Friedland. Autoresonant solutions of the nonlinear Schrödinger equation. Physical Review E, 58(3):3865-3875, Sept. 1998. 2

[30] L. Friedland and A. Shagalov. Emergence and Control of Multiphase Nonlinear Waves by Synchronization. Phys. Rev. Lett., 90(7):74101, Feb. 2003. 2

[31] J.-M. Ghidaglia, A. Kumbaro, and G. Le Coq. On the numerical solution to two fluid models via cell centered finite volume method. Eur. J. Mech. B/Fluids, 20:841-867, 2001. 9

[32] O. Glass and S. Guerrero. Some exact controllability results for the linear KDV equation and uniform controllability in the zero dispersion limit. Asymptot. Anal., 60(1/2):61-100, 2008. 3

[33] O. Glass and S. Guerrero. Controllability of the Korteweg-de Vries equation from the right Dirichlet boundary condition. Systems and Control Letters, 59(7):390-395, 2010. 3 
[34] J. Hammack. A note on tsunamis: their generation and propagation in an ocean of uniform depth. $J$. Fluid Mech., 60:769-799, 1973. 2

[35] A. Harten and S. Osher. Uniformly high-order accurate nonscillatory schemes. I. SIAM J. Numer. Anal., 24:279-309, 1987. 9

[36] S. L. Instant Sport. http://www.wavegarden.com/. 2012. 2, 18

[37] R. S. Johnson. A Modern Introduction to the Mathematical Theory of Water Waves. Cambridge University Press, 2004. 5

[38] D. J. Korteweg and G. de Vries. On the change of form of long waves advancing in a rectangular canal, and on a new type of long stationary waves. Phil. Mag., 39(5):422-443, 1895. 3

[39] B. M. Lake, H. C. Yuen, H. Rungaldier, and W. E. Ferguson. Nonlinear deep-water waves: theory and experiment. Part 2. Evolution of a continuous wave train. J. Fluid Mech, 83(01):49-74, Apr. 1977. 1

[40] C. Laurent, L. Rosier, and B.-Y. Zhang. Control and stabilization of the Korteweg-de Vries equation on a periodic domain. Comm. Partial Diff. Eqns., 35:707-744, 2010. 3

[41] S. Micu. On the Controllability of the Linearized Benjamin-Bona-Mahony Equation. SIAM Journal on Control and Optimization, 39(6):1677-1696, Jan. 2001. 4

[42] D. E. Mitsotakis. Boussinesq systems in two space dimensions over a variable bottom for the generation and propagation of tsunami waves. Math. Comp. Simul., 80:860-873, 2009. 18

[43] M. A. Nosov and S. V. Kolesov. Method of specification of the initial conditions for numerical tsunami modeling. Moscow University Physics Bulletin, 64(2):208-213, May 2009. 2

[44] M. A. Nosov and S. N. Skachko. Nonlinear tsunami generation mechanism. Natural Hazards and Earth System Sciences, 1:251-253, 2001. 2

[45] E. A. Okal and C. E. Synolakis. A theoretical comparison of tsunamis from dislocations and landslides. Pure and Applied Geophysics, 160:2177-2188, 2003. 2

[46] D. H. Peregrine. Long waves on a beach. J. Fluid Mech., 27:815-827, 1967. 3, 4

[47] G. Perla-Menzala, C. F. Vasconcellos, and E. Zuazua. Stabilization of the Korteweg-de Vries equation with localized damping. Quart. Appl. Math., 60:111-129, 2002. 3

[48] L. S. Pontryagin. Mathematical Theory of Optimal Processes. CRC Press, english ed edition, 1987. 3

[49] L. Rosier. Exact boundary controllability for the Korteweg-de Vries equation on a bounded domain. ESAIM Cntrol Optim. Calc. Var., 2:33-55, 1997. 3

[50] L. Rosier. Exact Boundary Controllability for the Linear Korteweg-de Vries Equation on the Half-Line. SIAM Journal on Control and Optimization, 39(2):331-351, Jan. 2000. 4

[51] L. Rosier. Control of the surface of a fluid by a wavemaker. ESAIM Cntrol Optim. Calc. Var., 10:346380, 2004. 3

[52] L. Rosier and B.-Y. Zhang. Unique continuation property and control for the Benjamin-Bona-Mahony equation on the torus. Arxiv, 1202.2667:35, 2012. 3

[53] L. Russell and B.-Y. Zhang. Controllability and stabilizability of the third-order linear dispersion equation on a periodic domain. SIAM Journal on Control and Optimization, 31:659-676, 1993. 3

[54] L. Russell and B.-Y. Zhang. Smoothing and decay properties of the Korteweg-de Vries equation on a periodic domain with point dissipation. J. Math. Anal. Appl., 190:449-488, 1995. 3

[55] L. Russell and B.-Y. Zhang. Exact Controllability and stabilizability of the Korteweg-de Vries equation. Trans. Amer. Math. Soc., 348:3653-3672, 1996. 3

[56] L. F. Shampine and M. W. Reichelt. The MATLAB ODE Suite. SIAM Journal on Scientific Computing, 18:1-22, 1997. 9

[57] S. Tadepalli and C. E. Synolakis. The run-up of N-waves on sloping beaches. Proc. R. Soc. Lond. A, 445:99-112, 1994. 14

[58] S. Tadepalli and C. E. Synolakis. Model for the leading waves of tsunamis. Phys. Rev. Lett., 77:21412144, 1996. 14

[59] Y. Tanioka and K. Satake. Tsunami generation by horizontal displacement of ocean bottom. Geophysical Research Letters, 23:861-864, 1996. 2

[60] M. I. Todorovska, A. Hayir, and M. D. Trifunac. A note on tsunami amplitudes above submarine slides and slumps. Soil Dynamics and Earthquake Engineering, 22:129-141, 2002. 2 
[61] M. I. Todorovska and M. D. Trifunac. Generation of tsunamis by a slowly spreading uplift of the seafloor. Soil Dynamics and Earthquake Engineering, 21:151-167, 2001. 2

[62] J. Touboul. Controllability of the heat and wave equations and their finite difference approximations by the shape of the domain. Mathematical Control and Related Fields, 2(4):429-455, Oct. 2012. 4

[63] F. Ursell. The long-wave paradox in the theory of gravity waves. Proc. Camb. Phil. Soc., 49:685-694, 1953. 3,5

[64] S. N. Ward. Landslide tsunami. J. Geophysical Res., 106:11201-11215, 2001. 2

[65] P. Watts, S. T. Grilli, J. T. Kirby, G. J. Fryer, and D. R. Tappin. Landslide tsunami case studies using a Boussinesq model and a fully nonlinear tsunami generation model. Natural Hazards And Earth System Science, 3(5):391-402, 2003. 2

[66] T. Y. T. Wu. Generation of upstream advancing solitons by moving disturbances. Journal of Fluid Mechanics, 184:75-99, 1987. 3, 4, 5

[67] B.-Y. Zhang. Exact boundary controllability of the Korteweg-de Vries equation. SIAM Journal on Control and Optimization, 37:543-565, 1999. 3

[68] B.-Y. Zhang, L. Rosier, J. Ortega, and S. Micu. Control and stabilization of a family of Boussinesq systems. Discrete and Continuous Dynamical Systems, 24(2):273-313, Mar. 2009. 4

[69] X. Zhang and E. Zuazua. Unique continuation for the linearized Benjamin-Bona-Mahony equation with space-dependent potential. Mathematische Annalen, 325(3):543-582, Mar. 2003. 4

BCAM - The Basque Center for Applied Mathematics, Alameda Mazarredo 14, 48009

Bilbao, Basque Country - Spain

E-mail address: hnersisyan@bcamath.org

URL: http://www.bcamath.org/en/people/nersisyan/

University College Dublin, School of Mathematical Sciences, Belfield, Dublin 4, IreLAND And LAMA, UMR 5127 CNRS, Université de Savoie, Campus Scientifique, 73376 Le Bourget-du-Lac Cedex, France

E-mail address: Denys.Dutykh@univ-savoie.fr

$U R L:$ http://www.denys-dutykh.com/

BCAM - The Basque Center for Applied Mathematics, Alameda Mazarredo 14, 48009 Bilbao, Basque Country - Spain and Ikerbasque, Basque Foundation for Science Alameda Urquijo 36-5, Plaza Bizkaia 48011, Bilbao, Basque Country, Spain

E-mail address: zuazua@bcamath.org

$U R L$ : http: //www . bcamath.org/en/people/zuazua/ 